\title{
Pregnancy Outcomes Following the First Frozen Blastocyst Transfer among Women Aged Less than 35 Years Old
}

\section{Le Hoang}

General Hospital

Le Duc Thang

general hospital

Nguyen Thi Lien Huong

General Hospital

Nguyen Minh Thuy

General Hospital

\section{Vu Thi Mai Anh}

General Hospital

Nguyen Thanh Duc

General Hospital

Nguyen Thi Dieu Chi

General Hospital

Tham Chi Dung

Ministry of Health and Child Care

jean-noël hugues ( $\nabla$ jnhugues@yahoo.fr)

Paris 13 University: Universite Sorbonne Paris Nord https://orcid.org/0000-0002-9607-6645

\section{Research}

Keywords: In-Vitro Fertilization, frozen blastocyst transfer, single embryo transfer, double embryo transfer, pregnancy, multiple pregnancy

Posted Date: March 2nd, 2021

DOI: https://doi.org/10.21203/rs.3.rs-247014/v1

License: (c) (1) This work is licensed under a Creative Commons Attribution 4.0 International License.

Read Full License 


\section{Abstract}

Aims of the study were to describe main outcomes (clinical, ongoing single and multiple pregnancy and live birth rates) following frozen blastocyst transfer performed for the first time among women aged less than 35 years old and analyzed according to both quantity and quality of the embryos. A descriptive cross-sectional study was applied to collect and analyze available data of 505 patients who performed transfer of frozen blastocysts for the first time between June, 2018 and September, 2019 at the Assisted Reproductive Technology Centre of Tam Anh General Hospital. One good quality embryo was transferred for 121 patients (Group 1), 2 good quality embryos for 214 patients (Group 2), 1 good and 1 poor quality embryo for 112 patients (Group 3), 1 good and 2 poor quality embryos for 25 patients (Group 4) and 1 or 2 poor quality embryos for 33 patients (Group 5). Main results showed that the pregnancy rate was $71.9 \%, 74.8 \%, 69.4 \%, 84.0 \%$ and $39.4 \%$ in Group $1,2,3,4$ and 5 , respectively. The rate of multiple pregnancy was $36.9 \%, 16.9 \%$, and $32.0 \%$ in Group 2, 3, and 4, respectively, higher than in Group 1 (4.9\%). Meanwhile, the live birth rate was 55.6\%,50.9\%, and $60.0 \%$ in Group 2, 3 and 4, respectively, but not significantly different from the live birth rate in Group 1 (47.9\%). In conclusion, pregnancy and live birth rates were not significantly different following transfer of 1 or 2 good quality blastocysts while the rate of multiple pregnancy was significantly increased following the transfer of 2 good quality ones. Transfer of 1 or 2 poor quality embryos in addition to 1 good embryo did not significantly improve the pregnancy rate.

\section{Introduction}

Since the success of the first frozen-stored embryo transfer (FET) in the world in 1983 [1], FET technique has become more popular and is now widely applied in many countries, including Vietnam. Frozen embryo transfer minimizes the risk of ovarian hyper-stimulation by reducing the potential negative effects of high concentration of reproductive hormones on the endometrium following the ovarian stimulation [2]. Two important indicators have been identified for selecting embryos for subsequent transfer: the quantity and quality of the embryos [3].

While the freezing of embryos was firstly performed at a cleavage stage, several improvements in lab materials such as incubators, culture media and quality control systems, allowed to get a blastocyst stage in a higher proportion of cleaved embryos.

Consequently, blastocyst transfer has become increasingly popular in clinical practice due to the ability to select the most developmentally competent embryo for transfer and to improve pregnancy rate. In order to develop to the blastocyst stage, embryos have to undergo a selection process during which lower quality embryos would stop growing and therefore would not be selected for transfer. In patients with many embryos, the strategy of freezing with subsequent transfer of frozen embryos has allowed to achieve a high cumulative pregnancy rate [2][4]. 
In an effort to prevent multiple pregnancy, the strategy of single embryo transfer (SET) has been recommended in clinical practice because it allows to achieve a live birth rate very close to that of double embryo transfer (DET) (38.0\% versus $42.0 \%)$ while reducing sharply the multiple pregnancy rate $(1.0 \%$ versus $32.0 \%$ ) [5]. Similar results have been recently published in a Cochrane review showing that the live birth rate in the SET group (27.0 - 35.0\%) was comparable to that of DET group (46.0\%) while the rate of multiple pregnancy was significantly different (2.0\% in SET group compared to $15.0 \%$ in DET group) [6].

As compared to single pregnancy, multiple pregnancy, a consequence of multiple embryos transfer, is associated with an increased incidence of cerebral palsy (3.2\% versus $2.5 \%)$, preterm birth $(6.0 \%$ versus $<1.0 \%)$, and low birth weight (24.0\% versus $8.0 \%)$ [5][7].

In addition, a study on the cost-effectiveness of DET versus SET after 2 consecutive cycles showed that DET is less cost-effective due to additional costs of managing short and long-term complications of multiple pregnancy [8]. Consequently, the Human Fertility and Embryology Authority (HEFA) recommended to get the objective of a low rate (below 10.0\%) of multiple pregnancy [9]. Nevertheless, DET is still acceptable for patients and health care workers when only embryos of poor quality are available for transfer or when one good quality embryo has been obtained in conjunction with another of poor quality.

Since 2000s, many studies comparing pregnancy outcomes according to the number of transferred embryos have provided conflicting results in pregnancy rate and multiple pregnancy rate. However, the scientific conclusion of these studies was limited by their usual small sample size, the heterogeneity of included subjects and in embryo quality assessment and, finally, the inconstant report of live birth rate [10]-[14].

In Vietnam, the overall cost of In-Vitro Fertilization (IVF) is not covered by medical insurance and the government has not yet implemented a policy to control the number of transferred embryos. Hence, couples have the opportunity to decide the number of embryos to be transferred after being informed about the benefits and risks of their decision. Meanwhile, the technique of frozen blastocyst transfer is widely applied in clinical practice but very few studies on the pregnancy outcome after freezing have been published so far [3]. Therefore, both medical staff and couples do lack actual medical information to take the optimal decision.

This study was conducted to analyze the outcome after transferring frozen blastocysts according to their quantity and quality among patients under 35 years old who performed their first embryo transfer in Tam Anh General Hospital during the period of 2018-2019. Purpose of this study was to provide evidencebased benefits and risks when deciding on the number and quality of transferred frozen-thawed blastocyst.

\section{Patients And Methods}

\section{Patients}


This study included women who had performed frozen blastocyst transfer at the Assisted Reproductive Technology (ART) Center, Tam Anh General Hospital within a 16-month period from June 2018 to September 2019. In this population, the following selection criteria were applied: patients $\leq 35$ years old at the time of ovarian stimulation for IVF or Intracytoplasmic Sperm Injection (ICSI), GnRH antagonist protocol, cryopreserved embryo transfer for the first time and use of autologous oocytes. Exclusion criteria were the following: patients with obstetrical and gynecological diseases (uterine fibroid, endometriosis, hydrosalpinx) and requirement of pre-implantation genetic testing.

\section{Procedures}

Ovarian stimulation for IVF: A standard GnRH antagonist protocol was applied in all patients. The starting dose of FSH was 150-300 IU/day (Gonal F/Follitrope/Puregon) with subsequent adjustment at day 6. A GnRH antagonist (Cetrorelix $0.25 \mathrm{mg} /$ day; Cetrotide, EMD Serono) was initiated at day 6th of ovarian stimulation. Final oocyte maturation was triggered by hCG 5000 IU (Pregnyl, Merck Sharp \& Dohme Asia, Vietnam) when the largest follicle reached $18 \mathrm{~mm}$ in diameter. Oocyte retrieval was performed 36 hours later and oocytes were fertilized using IVF/ICSI method.

Following in vitro culture, blastocysts were obtained and graded according to Gardner and Schoolcraft classification based on embryonic development stage (compression phase, early blastocyst, complete blastocyst, hatching blastocyst), trophoblast and inner cell mass morphology. An embryo was considered as good when graded $A A, A B, B A$ and $B B$. Poor quality embryos were blastocysts classified as $A C, C A, B C$, $\mathrm{CB}$, and $\mathrm{CC}[15],[16]$.

Blastocysts were frozen on day 5 or 6 by vitrification, using the CryoTec system (Vitrification kit 101, Reprolife, Japan), including sterile plates, cryotops and vitrification media. The number of embryos stored on a top depends on the embryo's quality and quantity along with the patient's history, with a maximum of three per top. To minimize the risk of multi-pregnancy, good quality embryos were recommended to be preserved one per top for the first two or three tops. In cases none or just a few good quality embryos were available, poor quality embryos were considered to be preserved with or without the good ones, and two to three embryos per top was allowed. Of all cases when more than one embryo was stored on a top, the couple was fully informed about the risk of multiple pregnancy and the possibility of embryo degeneration after thawing (if any poor embryo presented).

For subsequent transfer, an artificial cycle was used for endometrium preparation with consecutive hormonal regimen: estradiol valerate (Progynova, Bayer) $6 \mathrm{mg} /$ day starting on day 2 or 3 of the menstrual cycle. When the endometrial thickness was $>7 \mathrm{~mm}$, progesterone supplementation was added with Dydrogesterone $30 \mathrm{mg} /$ day (Dusphaton ${ }^{\circledR} 10 \mathrm{mg}$, Abott, t.i.d. orally) and vaginal suppository microProgesterone 600 mg/day (Utrogestan 200 mg, Besins Healthcare, b.i.d). Embryo(s) was transferred on day 6 th following progesterone supplementation. The number of transferred embryos was determined by the couple after being advised by an infertility specialist on the associated benefits and risks. 


\section{Statistics}

The measured variables included: Age in years; Body Mass Index (BMI) calculated with following formula: [BMI=Weight/[(Height) $\left.{ }^{2}\right]$ with height in meters and weight in kilograms; AMH (Anti-Mullerian Hormone) expressed in $\mathrm{ng} / \mathrm{ml}$. The endometrial thickness on the day of transfer was measured in millimeter. Infertility duration was counted in years.

Data was collected based on medical records and patients telephoned information, then encrypted and stored in our center's database. The necessary variable fields were exported for analysis with patients' name encoded.

The variables labeled from Epi-Data files were transferred into SPSS format (SPSS ver. 21.0) also rechecked using the codebook syntax to ensure the accuracy of the labels. SPSS software (SPSS ver. 21.0) was used for data analysis such as percentage, frequency, mean, standard deviation (SD), ANOVA test and Z-test in the statistical two-sample mean and proportion comparison at the confident level of $5 \%$. In order to assess the impact of embryo transfer policy on the outcome following frozen-thawed blastocysts transfer, the overall pregnancy rates were analyzed according to the number and the quality of transferred blastocysts. For that purpose, patients were separated into 5 groups defined as follows: Group 1: 1 good quality embryo transferred, Group 2: 2 good quality embryos transferred, Group 3: 1 good and 1 poor quality embryos transferred, Group 4: 1 good and 2 poor quality embryos transferred and Group 5: 1 or 2 poor quality embryos transferred (Figure 1).

Outcomes

The primary outcome was live birth rate defined as the deliveries of any viable neonate, expressed per 100 embryo transfers.

In addition, secondary efficacy outcomes included biochemical pregnancy rate (number of beta-hCG > 10 $\mathrm{mlU} / \mathrm{mL}$ cases /100 embryo transfers), clinical pregnancy rate (number of pregnancies with gestational sac per 100 embryo transfers), ongoing pregnancy rate (number of pregnancies that completed $\geq 20$ weeks of gestation per 100 embryo transfers). The multiple pregnancy rate was calculated as the percentage of pregnant women with two or more gestational sacs or positive heart beats by transvaginal sonography at 5 weeks after embryo placement per 100 transfers. The rate of ectopic pregnancy was the number of ectopic pregnancies (diagnosed by ultrasound, surgery or histopathology) /100 embryo transfers. The miscarriage rate was the number of spontaneous loss of pregnancy before $22^{\text {nd }}$ week gestational / 100 cases of embryo transfer. The preterm birth rate before the $32^{\text {nd }}$ week was the number of deliveries before the $32^{\text {nd }}$ week / 100 transfers.

\section{Results}

\section{Subject characteristics}


A total of 505 patients fulfilling the inclusion criteria and performing an IVF cycle from June 2018 to September 2019 were included in this analysis. These subjects were separated into the 5 different groups previously defined according to the following distribution: 121 patients in Group 1, 214 in Group 2, 112 in Group 3, 25 in Group 4, and 33 in Group 5 (Table 1).

Table 1

Subject characteristics according to 5 groups of frozen-thawed embryos transfers

\begin{tabular}{|c|c|c|c|c|c|c|}
\hline Characteristics & $\begin{array}{l}\text { Group } \\
1\end{array}$ & Group 2 & $\begin{array}{l}\text { Group } \\
3\end{array}$ & $\begin{array}{l}\text { Group } \\
4\end{array}$ & $\begin{array}{l}\text { Group } \\
5\end{array}$ & Total \\
\hline Number of subjects (n) & 121 & 214 & 112 & 25 & 33 & 505 \\
\hline \multirow[t]{2}{*}{ Age (year, 95\%Cl) } & 28.5 & 28.5 & 28.6 & 28.8 & 29.4 & 28.6 \\
\hline & $\begin{array}{l}(27.9- \\
29.2)^{a}\end{array}$ & $\begin{array}{l}(28.1- \\
28.9)^{b}\end{array}$ & $\begin{array}{l}(27.9- \\
29.1)^{b}\end{array}$ & $\begin{array}{l}\left(27.3^{-}\right. \\
30.2)^{b}\end{array}$ & $\begin{array}{l}(28.4- \\
30.5)^{b}\end{array}$ & $\begin{array}{l}(28.3- \\
28.9)\end{array}$ \\
\hline \multirow[t]{2}{*}{ BMI $\left(\mathrm{kg} / \mathrm{m}^{2}\right)$ (index, 95\%Cl) } & 21.1 & 21.2 & 21.4 & 21.2 & 20.9 & 21.2 \\
\hline & $\begin{array}{l}(20.7- \\
21.6)^{\mathrm{a}}\end{array}$ & $\begin{array}{l}(20.9- \\
21.5)^{\mathrm{b}}\end{array}$ & $\begin{array}{l}(20.9- \\
21.9)^{\mathrm{b}}\end{array}$ & $\begin{array}{l}(20.1- \\
22.4)^{b}\end{array}$ & $\begin{array}{l}(20.1- \\
21.7)^{\mathrm{b}}\end{array}$ & $\begin{array}{l}(21.0- \\
21.4)\end{array}$ \\
\hline \multirow{2}{*}{$\begin{array}{l}\text { Endometrium thickness (mm) } \\
\text { (thickness, } 95 \% \mathrm{Cl} \text { ) }\end{array}$} & 9.6 & 9.7 & 9.5 & 9.4 & 9.6 & 9.6 \\
\hline & $\begin{array}{l}(9.4- \\
9.8)^{a}\end{array}$ & $\begin{array}{l}(9.6- \\
9.9)^{b}\end{array}$ & $\begin{array}{l}\left(9.3^{-}\right. \\
9.7)^{b}\end{array}$ & $\begin{array}{l}(8.9- \\
9.9)^{\mathrm{b}}\end{array}$ & $\begin{array}{l}(9.2- \\
10.1)^{b}\end{array}$ & $\begin{array}{l}(9.5- \\
9.7)\end{array}$ \\
\hline \multirow[t]{2}{*}{ AMH (ng/ml) (index, 95\%Cl) } & 4.3 & 4.1 & 4.2 & 3.9 & 3.7 & 4.1 \\
\hline & $\begin{array}{l}(3.8- \\
4.7)^{\mathrm{a}}\end{array}$ & $\begin{array}{l}(3.7- \\
4.6)^{b}\end{array}$ & $\begin{array}{l}(3.6- \\
4.7)^{b}\end{array}$ & $\begin{array}{l}\left(2.8^{-}\right. \\
5.1)^{b}\end{array}$ & $\begin{array}{l}(2.9- \\
4.6)^{b}\end{array}$ & $\begin{array}{l}(3.9- \\
4.4)\end{array}$ \\
\hline \multirow{2}{*}{$\begin{array}{l}\text { Infertility duration (year) (years, } \\
95 \% \mathrm{Cl})\end{array}$} & 3.1 & 3.4 & 3.5 & 3.3 & 3.9 & 3.4 \\
\hline & $\begin{array}{l}\left(2.8^{-}\right. \\
3.5)^{\mathrm{a}}\end{array}$ & $\begin{array}{l}(3.1- \\
3.7)^{b}\end{array}$ & $\begin{array}{l}(3.1- \\
3.9)^{b}\end{array}$ & $\begin{array}{l}\left(2.5^{-}\right. \\
4.2)^{b}\end{array}$ & $\begin{array}{l}(3.2- \\
4.7)^{\mathrm{b} *}\end{array}$ & $\begin{array}{l}(3.2- \\
3.6)\end{array}$ \\
\hline Primary infertility $(\mathrm{n}, \%)$ & $\begin{array}{l}95 \\
(78.5)^{\mathrm{a}}\end{array}$ & $\begin{array}{l}186 \\
(86.9)^{b \star \star}\end{array}$ & $\begin{array}{l}96 \\
(85.7)^{b}\end{array}$ & $\begin{array}{l}19 \\
(76.0)^{b}\end{array}$ & $\begin{array}{l}28 \\
(84.9)^{b}\end{array}$ & $\begin{array}{l}424 \\
(83.9)\end{array}$ \\
\hline \multicolumn{7}{|c|}{ Note: ${ }^{a}$ Group 1 was the reference group; ${ }^{b}$ Compared with reference group (Group 1); } \\
\hline \multicolumn{7}{|c|}{${ }^{*}$ ANOVA test with $p<0.05 ; * * Z$-test with $p<0.05$} \\
\hline
\end{tabular}

The mean age of all subjects was 28.6 years old (95\% Cl: $28.3-28.9)$, with an average age of 28.5 (95\% Cl: 27.9-29.2) in Group 1, not statistically different from other groups.

The mean duration of infertility was 3.4 years $(95 \% \mathrm{Cl}$ : 3.2-3.6), significantly longer in Group 5 (3.9 years, 95\% Cl: 3.2-4.7) than in Group 1 (3.1 years, 95\% Cl: 2.8-3.5) (ANOVA test, $\mathrm{p}<0.05$ ). 
Primary infertility accounted for $83.9 \%(424 / 505)$ with the highest rate in Group $2(86.9 \% ; 186 / 214)$, significantly different compared to Group 1 (Z-test, $p<0.05)$

The average BMI was $21.2 \mathrm{~kg} / \mathrm{m}^{2}$ (95\% Cl: 21.0-21.4). The average endometrium thickness was $9.6 \mathrm{~mm}$ (95\%Cl: 9.5-9.7); The mean AMH was $4.1 \mathrm{ng} / \mathrm{ml}(95 \% \mathrm{Cl}$ : 3.9-4.4) without any significant difference between Group 1 and other groups ( $p>0.05)$.

\section{Pregnancy outcomes according to embryo Groups}

Table 2 indicates that the overall pregnancy rate was $71.1 \%$ (359/505), with the highest pregnancy rate in Group $2(74.8 \% ; 160 / 214)$ and the lowest in Group $5(39.3 \% ; 13 / 33)$.

Table 2

Pregnancy outcomes according to 5 frozen embryo transfer Groups

\begin{tabular}{|c|c|c|c|c|c|c|}
\hline $\begin{array}{l}\text { Pregnancy outcomes after } \\
\text { embryo transfer }\end{array}$ & $\begin{array}{l}\text { Group } \\
1\end{array}$ & Group 2 & Group 3 & $\begin{array}{l}\text { Group } \\
4\end{array}$ & Group 5 & Total \\
\hline Number of subjects (n) & 121 & 214 & 112 & 25 & 33 & 505 \\
\hline Pregnancy $(n, \%)$ & $\begin{array}{l}87 \\
(71.9)^{a}\end{array}$ & $\begin{array}{l}160 \\
(74.8)^{\mathrm{b}}\end{array}$ & $\begin{array}{l}78 \\
(69.4)^{b}\end{array}$ & $\begin{array}{l}21 \\
(84.0)^{b}\end{array}$ & $\begin{array}{l}13 \\
(39.4)^{b}\end{array}$ & $\begin{array}{l}359 \\
(71.1)\end{array}$ \\
\hline Clinical pregnancy $(n, \%)$ & $\begin{array}{l}69 \\
(57.0)^{a}\end{array}$ & $\begin{array}{l}146 \\
(68.2 \%)^{\mathrm{b} *}\end{array}$ & $\begin{array}{l}67 \\
(59.8)^{b}\end{array}$ & $\begin{array}{l}17 \\
(68.0)^{b}\end{array}$ & $\begin{array}{l}10 \\
(30.3) \\
b_{*}\end{array}$ & $\begin{array}{l}309 \\
(61.2)\end{array}$ \\
\hline Ongoing pregnancy $(n, \%)$ & $\begin{array}{l}58 \\
(47.9)^{a}\end{array}$ & $\begin{array}{l}119 \\
(55.6)^{b}\end{array}$ & $\begin{array}{l}57 \\
(50.9)^{b}\end{array}$ & $\begin{array}{l}15 \\
(60.0)^{b}\end{array}$ & $\begin{array}{l}6(18.2) \\
b *\end{array}$ & $\begin{array}{l}255 \\
(50.5)\end{array}$ \\
\hline Live birth $(n, \%)$ & $\begin{array}{l}58 \\
(47.9)^{a}\end{array}$ & $\begin{array}{l}119 \\
(55.6)^{\mathrm{b}}\end{array}$ & $\begin{array}{l}57 \\
(50.9)^{b}\end{array}$ & $\begin{array}{l}15 \\
(60.0)^{b}\end{array}$ & $\begin{array}{l}6(18.2) \\
b *\end{array}$ & $\begin{array}{l}255 \\
(50.5)\end{array}$ \\
\hline Multiple pregnancy $(n, \%)$ & $\begin{array}{l}6 \\
(4.9)^{a}\end{array}$ & $\begin{array}{l}79(36.9) \\
b_{*}\end{array}$ & $\begin{array}{l}19 \\
(16.9) \\
b *\end{array}$ & $\begin{array}{l}8 \\
(32.0) \\
b_{*}\end{array}$ & $1(3.0)^{b}$ & $\begin{array}{l}113 \\
(22.4)\end{array}$ \\
\hline Preterm birth $<32^{\text {nd }}$ week $(n, \%)$ & $\begin{array}{l}2 \\
(1.7)^{a}\end{array}$ & $10(4.7)^{b}$ & $4(3.6)^{b}$ & $\begin{array}{l}3 \\
(12.0) \\
b_{\star}\end{array}$ & $0(0)^{b}$ & $\begin{array}{l}19 \\
(3.8)\end{array}$ \\
\hline Ectopic pregnancy $(n, \%)$ & $\begin{array}{l}2 \\
(1.7)^{\mathrm{a}}\end{array}$ & $3(1.4)^{b}$ & $2(1.8)^{b}$ & $\begin{array}{l}0(0.0) \\
b\end{array}$ & $0(0.0)^{b}$ & $7(1.4)$ \\
\hline Miscarriage $(n, \%)$ & $\begin{array}{l}29 \\
(23.9)^{a}\end{array}$ & $\begin{array}{l}41(19.2) \\
\mathrm{b}\end{array}$ & $\begin{array}{l}21 \\
(18.8)^{b}\end{array}$ & $\begin{array}{l}6 \\
(24.0)^{b}\end{array}$ & $\begin{array}{l}7(21.2) \\
\mathrm{b}\end{array}$ & $\begin{array}{l}104 \\
(20.6)\end{array}$ \\
\hline
\end{tabular}


Regarding the overall clinical pregnancy rate $(61.2 \% ; 309 / 505)$, the highest was observed in Group 2 $(68.2 \% ; 146 / 214)$ and the lowest in Group $5(30.3 \% ; 10 / 33)$.

The overall live birth rate was $50.5 \%$ (255/505), with the highest rate in Group $4(60.0 \% ; 15 / 25)$, the lowest in Group $5(18.2 \%$; 6/33) which was significantly lower than in Group $1(47.9 \%)$ (Z-test, $p<0.05)$. No significant difference existed between Groups 2, 3, 4 and Group 1 (Z-test, $p>0.05$ ).

The overall multiple pregnancy rate was $22.4 \%$ (113/505), with the highest rate in Group 2 (36.9\%; $79 / 214)$, lowest in Group $5(3.0 \% ; 1 / 33)$. A significant increase in multiple pregnancy rate was found in Groups 2, 3 and 4 compared to Group 1 (Z-test, $p<0.05$ ).

The overall preterm birth rate before $32^{\text {nd }}$ week was $3.8 \%(19 / 505)$. There were 3 cases of preterm delivery reported in Group 4, accounting for $12.0 \%(3 / 25)$. This rate was low in other groups $(<5.0 \%)$.

In this study, the overall ectopic pregnancy rate was $1.4 \%$ (7/505), not significant in Group 1,2 and 3 and this condition did not occur in Group 4, 5.

A total of 104 miscarriages was reported, accounting for $20.6 \%$ of the overall pregnancies. Miscarriage occurred mainly in Group $4(24.0 \% ; 6 / 25)$ and in Group $1 .(23.9 \% ; 29 / 121)$ but the difference was not significant (Z-test, $p>0.05)$.

\section{Discussion}

This analysis of data collected from more than 5 hundred patients allows us to conclude that transfer of a single good frozen blastocyst leads to pregnancy and live birth rates similar to that observed after transfer of two good embryos but results in significantly lower multiple pregnancy potentiality. Additionally, transfer of 1 or 2 poor quality embryos along with 1 good embryo does not increase the chance of pregnancy.

The population included in this study was selected for the absence of previous pelvic pathology (uterine fibroid, endometriosis, hydrosalpinx). Main characteristics of these 5 groups of patients (age, BMI, ovarian reserve, responsiveness to hormones) attest that their prognosis of pregnancy was presumably good.

The infertility duration was slightly higher in Group 5, being 3.9 years $(95 \% \mathrm{Cl} 3.2-4.7)$, compared to other groups. This could be related to the poor quality of the blastocyst.

The rate of primary infertility was $86.9 \%$ in Group 2 , significantly different from Group 1 . This difference might well-explain their desire to take the risk of multiple pregnancy with transfer of 2 good quality embryos. Even if couples have the right to decide the strategy for embryo transfer, the medical staff should advise them regarding benefits and risks of transferring 2 embryos. As the risk of multiple pregnancy was not widely known, the rate of transferring two good embryos in the primary infertility 
group remained high. Our analysis provides evidence to recommend the transfer of a single embryo in those patients with a good fertility prognosis.

It is the embryo quality that is the major factor affecting pregnancy outcomes. Accordingly, patients in Group 5 with embryo(s) of poor quality displayed a significantly lower pregnancy rate compared to Groups 1 or 2. In contrast, the number of embryos transferred in the limit of 2 or 3 does not seem to be a main determinant of pregnancy rate. Indeed, no difference actually existed in pregnancy rates between Groups 2, 3, 4 and Group 1. The study by Dobson showed similar results [12] while the pregnancy rate in our series was higher presumably because our subjects had much lower age and performed frozen embryos transfer for the first time.

The risk of multiple pregnancy is another important issue to be addressed. It clearly exists when more than 1 blastocyst is transferred. In our study, the multiple pregnancy rate was significantly higher in Groups 2, 3, 4 than Group 1. It was about half of pregnant patients in Group 2 that experienced multiple pregnancy (79/159). In Dobson's publication, the difference was observed with a multiple pregnancy rate of $2.1 \%$ in the group transferring 1 good embryo and $16.6 \%$ in the group transferring 2 good embryos [12]. In our study, the multiple pregnancy rate of single blastocyst transfer was $4.9 \%$, while in others it was usually $2-3 \%$ [17], [18]. This may be due to the limitation of our sample size as well as differences in definition of multiple pregnancy, and in micromanipulation procedures (ICSI, assisted hatching) between studies.

Transferring of 1 good and 1 poor quality embryo resulted in similar pregnancy rate as compared to a single good embryo transfer (69.9\% versus $71.1 \%)$. However, the rate of multiple pregnancy was significantly higher (16.9\% versus $4.9 \%)$. Therefore, when the endometrial environment is favorable, a double embryo transfer (DET) with one good and one poor embryo can still lead to twin pregnancy. Our results are similar to those reported by Dobson et al., the transfer of 1 or more poor quality embryos in addition to a good quality embryo could result in higher multiple pregnancy rate (7.1\% versus $2.1 \%)$ [12].

Previous studies have suggested that interactions between an embryo and the endometrium during implantation may affect the implantation of other embryos. Therefore, when performing transfer of poor embryo(s), the endometrium can change to create an unfavorable environment for any accompanying good embryo's development, resulted in reduced pregnancy rate [19], [20]. However, Hill et al. reported that the transfer of one good embryo with another poor quality embryo in patients younger than 38 years resulted in an increase by $7 \%$ of live birth rate, but with a concomitant increase in multiple pregnancy rate by $18 \%$ [21]. In clinical practice, when only 2 embryos (1 good and 1 poor quality) are available, the couple and the health worker often decide to transfer both embryos in a single transfer. The common objective is clearly to increase pregnancy rate at the first transfer and to avoid the possibility of having only 1 poor quality embryo left for transfer if the first one failed. So, in that situation, these data could probably provide useful scientific evidence and incentive to change the clinical practice.

In this analysis, there was no statistically significant difference in live birth existed among Group 1, 2, 3 and 4, while the live birth rate in Group 5 was quite low (18.2\%, significantly lower than $47.9 \%$ in Group 1$)$. 
Indeed, poor quality embryos usually display higher chromosomal abnormality rate and low survival rate. Cimadomo et al. performed biopsies of 1,497 poor quality blastocyst and only 352 (23.5\%) were euploid, among them 195 were thawed, 193 survived and resulted, after transfer, in a low live birth rate (10.9\%). In contrast, $51 \%(2,680 / 5,250)$ of good quality embryos were euploid and resulted in the live birth rate of $44.8 \%(757 / 1,690)$ after single euploid good quality embryo transfer [22]. Therefore, a couple should be fully informed about the potential low success rate when transferring only poor-quality embryo.

In low-income countries, about half of babies born prematurely (before $32^{\text {nd }}$ week gestation) will die [23]. Therefore, limiting number of premature deliveries before $32^{\text {nd }}$ week of gestation is an important goal. The rate of preterm birth in Group 2 and 3 was higher than in Group 1 confirming the fact that multiple pregnancy is the leading risk for preterm delivery. According to statistics, the rate of preterm birth before 32 weeks in the single and twins group in the United States (2006) was 1.6\% and 13.3\% [24]. The difference in the preterm birth rate of our data may be due to the insufficient sample size and a more selected population (age of $\leq 35$, first transfer of embryos, few underlying diseases, few pregnancy diseases).

Several countries around the world have issued guidelines to ban the transfer of more than 2 embryos and even recommended the transfer of only 1 embryo into the mother's uterus [25], [26]. Therefore, IVF centers need to synchronously implement several measures in order to optimize the procedure of embryo culturing, to select the best quality embryo for transfer and to maintain high pregnancy rate per SET.

The limitation of this study was the retrospective cohort study design, using retrospective data collection method based on available medical records at a single IVF Center. The sample sizes of Group 4 and Group 5 were not large enough to detect statistical differences and be representative. Further in-depth study designs such as randomized controlled trial should be set to confirm the efficacy of single blastocyst transfer compared to transfer of multiple embryos.

\section{Conclusions}

Transfer of a single good quality frozen-thawed blastocyst leads to pregnancy and live birth rates similar to that of transferring two good quality embryos but with significant lower risk of multiple pregnancy. Hence, the transfer of more than one blastocyst should be avoided in patients transferring frozen embryos for the first time. Additionally, transfer of 1 or 2 poor quality embryos along with 1 good embryo does not improve the chance of pregnancy. These results could contribute to the change of clinical practice of embryo transfer strategy, especially in those countries of high multiple pregnancy rate following assisted reproductive treatment.

\section{Abbreviations}

FET: frozen-stored embryo transfer

SET: single embryo transfer 
DET: double embryo transfer

ART: Assisted Reproductive Technology

IVF: In-Vitro Fertilization

ICSI: Intracytoplasmic Sperm Injection

BMI: Body Mass Index

\section{Declarations}

\section{Ethics approval and consent to participate}

The research proposal was approved by the Scientific Research Council and Institutional Review Board of Tam Anh General Hospital under the Decision No. 85/QD-BVTA dated March 31, 2020 before initiating any research activities.

\section{Consent for publication}

All data and information were retrospectively collected based on electronic database, therefore the research did not cause any impacts on the patient's treatment. During the research, all patient individual information was absolutely guaranteed their confidentialities. Principal investigator was not allowed to collect any patient's personal information, but could only access to individual information through patient's identification number (PID) without any individual recognition.

\section{Availability of data and materials}

A dataset was used and analyzed for this study which was available and accessible through the corresponding author.

\section{Competing interests}

All authors declare that they have no competing interests.

\section{Funding}

This work was supported by the Tam Anh General Hospital. Open Access funding enabled and organized by Tam Anh General Hospital. 


\section{Authors' contributions}

Le Hoang and Le Duc Thang conceived and planned the clinical trial, analyzed data and wrote the manuscript. Le Duc Thang wrote the manuscript with support from Le Hoang, Nguyen Thi Lien Huong, Nguyen Minh Thuy, Vu Thi Mai Anh, Nguyen Thanh Duc. Nguyen Thi Dieu Chi helped supervise the project and data collection process. Tham Chi Dung wrote the manuscript and took part in data analysis. Jean-Noël Hugues contributed to the revision and intellectual content of this manuscript. All authors read and approved the final manuscript.

\section{Acknowledgments}

Authors sincerely thank the support, assistance and provision of data and related information of healthcare staff and all of patients in the Assisted Reproductive Technology Center of Tam Anh General Hospital.

\section{References}

1. A. Trounson and L. Mohr, "Human pregnancy following cryopreservation, thawing and transfer of an eight-Cell embryo," Nature, vol. 305, no. 5936, pp. 707-709, 1983, doi: 10.1038/305707a0.

2. C. Blockeel et al., "Should we still perform fresh embryo transfers in ART?," Hum. Reprod., vol. 34, no. 12, pp. 2319-2331, Dec. 2019, doi: 10.1093/humrep/dez233.

3. Q. Zhu, J. Lin, H. Gao, N. Wang, B. Wang, and Y. Wang, "The Association Between Embryo Quality, Number of Transferred Embryos and Live Birth Rate After Vitrified Cleavage-Stage Embryos and Blastocyst Transfer," Front. Physiol., vol. 11, p. 930, Aug. 2020, doi: 10.3389/fphys.2020.00930.

4. N. P. Polyzos et al., "Cumulative live birth rates according to the number of oocytes retrieved after the first ovarian stimulation for in vitro fertilization/intracytoplasmic sperm injection: a multicenter multinational analysis including $\sim 15,000$ women," Fertil. Steril., vol. 110, no. 4, pp. 661-670.e1, Sep. 2018, doi: 10.1016/j.fertnstert.2018.04.039.

5. D. J. McLernon et al., "Clinical effectiveness of elective single versus double embryo transfer: Metaanalysis of individual patient data from randomised trials," BMJ, vol. 342, no. 7787, p. 34, Jan. 2011, doi: 10.1136/bmj.c6945.

6. M. S. Kamath, M. Mascarenhas, R. Kirubakaran, and S. Bhattacharya, "Number of embryos for transfer following in vitro fertilisation or intra-cytoplasmic sperm injection," Cochrane Database of Systematic Reviews, vol. 2020, no. 8. John Wiley and Sons Ltd, Aug. 22, 2020, doi: 10.1002/14651858.CD003416.pub5.

7. S. Impact and P. No, "Multiple Pregnancies Following Assisted Conception: Scientific Impact Paper No. 22," BJOG An Int. J. Obstet. Gynaecol., vol. 125, no. 5, pp. e12-e18, 2018, doi: 10.1111/14710528.14974 . 
8. H. G. M. Lukassen et al., "Two cycles with single embryo transfer versus one cycle with double embryo transfer: A randomized controlled trial," Hum. Reprod., vol. 20, no. 3, pp. 702-708, 2005, doi: 10.1093/humrep/deh672.

9. "Our campaign to reduce multiple births," Human Fertilisation and Embryology Authority. https://www.hfea.gov.uk/about-us/our-campaign-to-reduce-multiple-births/.

10. D. K. Gardner, E. Surrey, D. Minjarez, A. Leitz, J. Stevens, and W. B. Schoolcraft, "Single blastocyst transfer: A prospective randomized trial," Fertil. Steril., vol. 81, no. 3, pp. 551-555, Mar. 2004, doi: 10.1016/j.fertnstert.2003.07.023.

11. T. A. Gelbaya, I. Tsoumpou, and L. G. Nardo, "The likelihood of live birth and multiple birth after single versus double embryo transfer at the cleavage stage: A systematic review and meta-analysis," Fertil. Steril., vol. 94, no. 3, pp. 936-945, Aug. 2010, doi: 10.1016/j.fertnstert.2009.04.003.

12. S. J. A. Dobson, M. T. Lao, E. Michael, A. C. Varghese, and K. Jayaprakasan, "Effect of transfer of a poor quality embryo along with a top quality embryo on the outcome during fresh and frozen in vitro fertilization cycles," Fertil. Steril., vol. 110, no. 4, pp. 655-660, Sep. 2018, doi:

10.1016/j.fertnstert.2018.05.010.

13. E. M. Wintner et al., "Does the transfer of a poor quality embryo together with a good quality embryo affect the In Vitro Fertilization (IVF) outcome?," J. Ovarian Res., vol. 10, no. 1, pp. 1-5, Jan. 2017, doi: 10.1186/s13048-016-0297-9.

14. R. L. R. Baruffi et al., "Single-embryo transfer reduces clinical pregnancy rates and live births in fresh IVF and Intracytoplasmic Sperm Injection (ICSI) cycles: A meta-analysis," Reproductive Biology and Endocrinology, vol. 7. Centre for Reviews and Dissemination (UK), Apr. 23, 2009, doi: 10.1186/14777827-7-36.

15. W. B. Schoolcraft, D. K. Gardner, M. Lane, T. Schlenker, F. Hamilton, and D. R. Meldrum, "Blastocyst culture and transfer: Analysis of results and parameters affecting outcome in two in vitro fertilization programs," Fertil. Steril., vol. 72, no. 4, pp. 604-609, 1999, doi: 10.1016/S0015-0282(99)00311-8.

16. T. Hardarson, L. Van Landuyt, and G. Jones, "The blastocyst," Hum. Reprod., vol. 27, no. suppl 1, pp. i72-i91, Aug. 2012, doi: 10.1093/humrep/des230.

17. T. Osianlis, L. Rombauts, M. Gabbe, C. Motteram, and B. Vollenhoven, "Incidence and zygosity of twin births following transfers using a single fresh or frozen embryo," Hum. Reprod., vol. 29, no. 7, pp. 1438-1443, 2014, doi: 10.1093/humrep/deu064.

18. J. R. Kanter, S. L. Boulet, J. F. Kawwass, D. J. Jamieson, and D. M. Kissin, "Trends and correlates of monozygotic twinning after single embryo transfer," Obstet. Gynecol., vol. 125, no. 1, pp. 111-117, Jan. 2015, doi: 10.1097/AOG.0000000000000579.

19. J. J. Brosens et al., "Uterine selection of human embryos at implantation," Sci. Rep., vol. 4, no. 1, pp. 1-8, Feb. 2014, doi: 10.1038/srep03894.

20. N. S. Macklon and J. J. Brosens, "The Human Endometrium as a Sensor of Embryo Quality," Biol. Reprod., vol. 91, no. 4, pp. 98-99, Oct. 2014, doi: 10.1095/biolreprod.114.122846. 
21. M. J. Hill et al., "Is transferring a lower-quality embryo with a good-quality blastocyst detrimental to the likelihood of live birth?," Fertil. Steril., vol. 114, no. 2, pp. 338-345, Aug. 2020, doi: 10.1016/j.fertnstert.2020.03.027.

22. D. Cimadomo et al., "Looking past the appearance: A comprehensive description of the clinical contribution of poor-quality blastocysts to increase live birth rates during cycles with aneuploidy testing," Hum. Reprod., vol. 34, no. 7, pp. 1206-1214, 2019, doi: 10.1093/humrep/dez078.

23. "Preterm birth." https://www.who.int/news-room/fact-sheets/detail/preterm-birth (accessed Oct. 14, 2020).

24. J. A. Martin et al., "National Vital Statistics Reports, Volume 57, Number 7, (January 7, 2009)," 2006. Accessed: Sep. 21, 2020. [Online]. Available: http://www.cdc.gov/nchs/VitalStats.htm.

25. A. O. F. Survey, "Chapter 5: Number of embryos for transfer in ART," Fertil. Steril., vol. 87, no. 4 SUPPL. 1, 2007, doi: 10.1016/j.fertnstert.2007.01.089.

26. R. Klitzman, "Deciding how many embryos to transfer: ongoing challenges and dilemmas," Reprod. Biomed. Soc. Online, vol. 3, pp. 1-15, 2016, doi: 10.1016/j.rbms.2016.07.001.

\section{Figures}

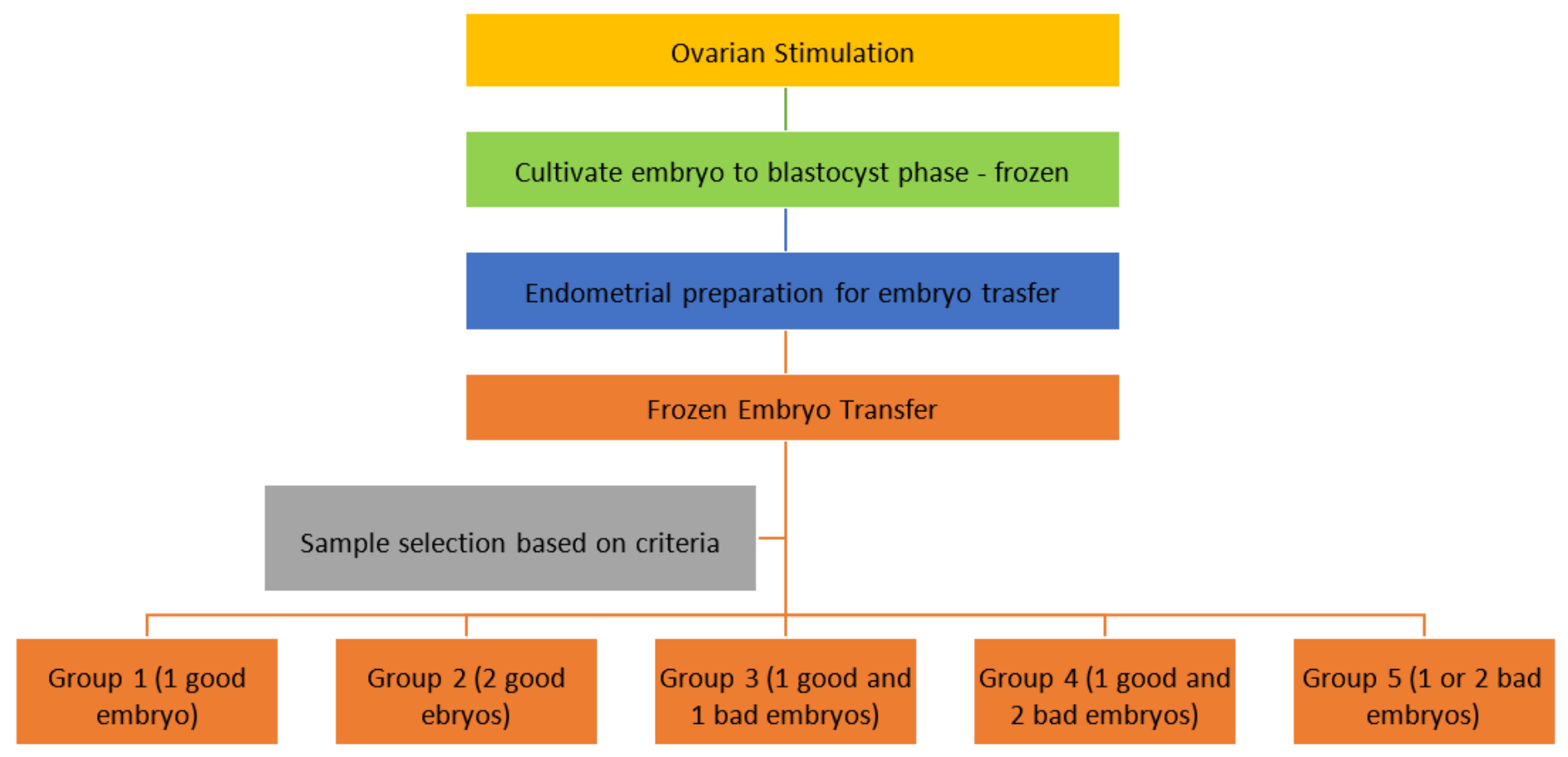

\section{Figure 1}

Frozen embryo transfer procedure 